\title{
Salivary Cortisol in an Extreme Non-Competitive Sport Exercise: Winter Swimming
}

\author{
Patrizia Loria' ${ }^{1}$, Stefano Ottoboni ${ }^{1}$, Luigi Michelazzi ${ }^{2}$, Roberto Giuria ${ }^{1}$, Paola Ghisellini' ${ }^{1}$, \\ Cristina Rando', Roberto Eggenhöffner ${ }^{1}$ \\ ${ }^{1}$ Department of Surgical Sciences and Integrated Diagnostics, University of Genoa, Genoa, Italy \\ ${ }^{2}$ Department of Health Sciences, University of Genoa, Genoa, Italy \\ Email: roberto.eggenhoffner@unige.it
}

Received 14 December 2013; revised 14 January 2014; accepted 21 January 2014

Copyright (C) 2014 by authors and Scientific Research Publishing Inc.

This work is licensed under the Creative Commons Attribution International License (CC BY).

http://creativecommons.org/licenses/by/4.0/

c) (i) Open Access

\section{Abstract}

Salivary cortisol role in response to strong stressors implied in extreme exercises and in sport practice was investigated with the aim to verify the claimed benefits that steers winter swimmers to self-prescribe the trials. Specific biochemical data allow to study a variety of stressors in sports and physical exercises, including extreme ones as winter swimming. Salivary cortisol behavior was examined in winter swimmers trials and canoe, canoe-polo competitions and comparisons of results between days with and without performances were reported. Cortisol circadian rhythm in sedentary subjects was collected as control. All the subjects were selected after anamnesticclinical checks to evaluate their physiological conditions. The circadian cortisol behavior was reported in days with competitions and trials as well as between these events. Abrupt cortisol concentration changes were detected at the time of the trials and competitions: surprisingly, large increasing and decreasing concentrations were detected in both groups. Moreover, in winter swimmers, cortisol concentration remained fairly elevated in the evening of the trial days. In days without competitions, the usual cortisol circadian rhythm was recovered in sportsmen whereas cortisol concentrations persisted at high levels up to the evening in winter swimmers. The view that an extreme sport-like exercise as the winter swimming may well pose some treats ranging from subclinical aspects up to dismetabolic pathologies and even cardiovascular risks is strengthened by results of cortisol trends, suggesting to check physiological conditions. Results demonstrate that well-being feeling can be in contrast to the claimed improvements of health.

\section{Keywords}

Stress Hormone, Winter Swimmers, Sportsmen, Adaptation Syndrome, Immunological Analysis 


\section{Introduction}

An overwhelming research interest has been devoted for the last years towards the physical exercise and its interrelationships with the neuroendocrine axis activity. The aim of the present research was to establish salivary cortisol role in response to strong stressors implied in extreme exercises and in sport practice. The biochemical assessment of salivary hormones is indeed the route to achieve a quantitative detection of human stress conditions in ordinary people. Such attainment would overcome qualitative evaluations of the stress employed so far for instance in working environment through questionnaires. Cortisol is regarded as the most important hormone controlling and signaling such activity [1]-[3] which is referred to within the general definition of "stress". Cortisol is traced in the saliva and salivary levels correlate with plasma cortisol concentrations. Actually, the concentration of cortisol in a salivary sample can be measured with the same reliability of its determination in blood [4]. The haematological parameters in winter swimmers show strong non pathological modifications [5]. Moreover, other hormones can be found in saliva for assessing stress beyond cortisol [6] [7]. As compared to plasma analysis, easy and not-invasive saliva collection represents a significant advantage to every research that necessarily involves very large number of samples [8] [9]. The sport or physical exercise differs from competitive sports practice because it is chosen autonomously and performed with the aim of improving own well-being, independently from sex, age, physical shape and from various physiological and pathological conditions. A growing amount of common people, practically every section of the population, is becoming involved in these activities, sometimes with extreme features, both for leisure activities and for a better health status. Many studies prove that cortisol concentration assumes an irregular trend [10] at the peak of the stressor effects on the organism. In this regard, both competitive sport activity and an extreme stressor trial are able to trigger the activity of the neuroendocrine axis. An uncompetitive physical exercise can highlight better the effects of an extreme stressor than a sport activity since the former lacks the emotional factors of a sport competition likely to affect the physiological cortisol rhythm.

The winter swimming trial (cimento in italian) is performed in many countries in freely accessible sea water in wintertime, also in adverse weather conditions, mostly in weekends along many Italian sea coasts. Water temperature ranges from $8^{\circ} \mathrm{C}$ to $14^{\circ} \mathrm{C}$. Swimmers are used to enter water all together, and they remain in water for a variable time not exceeding 20 minutes, without wearing wetsuit or protections against cold. The trials are closely monitored by medical protection and a security system. Obviously, as a counterpart of the extreme psychological and thermal stressor selected, only a rather limited number of practicing people can be found, actually a typical condition common to every extreme exercise. The present study assumes that people actively taking part in sporting exercise can experience a concentration of salivary cortisol not affected by psychological and physical pressures typically related to the competition and to the result of the sport contest. This is expected both in a day with or without trials or competitions. The latter occurs in the middle of the week days whereas trials/competitions in Sunday. Therefore, the behavior of cortisol in the winter swimming trials is compared in the present study with that of a group of canoe and canoe-polo sportsmen, both during the days of the competitive sport events and the trials [11] as well as in days without these performances.

\section{Materials and Methods}

\subsection{Winter Swimmers Subjects}

Subjects with long experience in winter swimming trial [12] were selected, ranging in age from 19 to 86 years, 7 females and 13 males. They were examined through an anamnestic-clinical check to evaluate their physiological conditions (sex, age, profession, and particular lifestyle like drinking, drugs and smoking), the basal parameters of the cardio respiratory apparatus (arterial blood pressure and pulse rate) and current diseases or taking of medicines (metabolic, respiratory, and chronic mental diseases). Each subjects were periodically checked and their medical chart were updated to exclude occurrences of new diseases [13] [14].

Exclusion criteria were histories of clinical manifestations of atherosclerosis (coronary artery, peripheral artery and cerebrovascular diseases), obesity, hypertension, diabetes mellitus, hyperlipidemia, venous thromboembolism, and liver or renal diseases. Exclusion criteria included also any routine medications, hormone therapy, or dietary supplements and also the practice of sports. The results concerning winter swimmer women in the starting group are not reported in the present work to avoid a further variable in the comparison with male sportsmen. The age of selected male winter swimmers ranges from 38 to 65 years, in the sportsmen from 22 to 
30 years. All selected winter swimmers performed five and four trials before and after the two examined ones, respectively, in the first months of 2011. The protocol applied in the study was approved by the University of Genoa, Italy, and all of the subjects provided their written fully informed consent. The ethical principles of medical associations and the ethical standards of the journal were fulfilled in agreement with the statements in [15] (ref. Harriss DJ 2011). Salivary samples were collected during two trials and in a day without winter swimming in the middle of the week.

\subsection{Sportsmen Subjects}

The same anamnestic-clinical protocol discussed above for the winter swimmers was applied to the group of five canoeists and four canoe-polo sportsmen. This control group consisting of sportsman was chosen for its consistency with the small number of winter swimmers selected to match strictly the clinical protocol. Actually it is not surprising that only small number of people is willing to undergo extreme stressor and that every investigations must face necessarily such fairly limited statistics. Anyway, the many papers referred to in the present work deals with comparable small numbers of selected participants.

\subsection{Sedentary Subjects}

Salivary samples were collected also from four sedentary subjects (from 40 to 60 years old) selected throughout the same protocol discussed above, carried out three times a day as from the above groups in days without events. The authors are well aware about the larger range of the age in the winter swimmers with respect to young sportsmen; however, this is an intrinsic characteristic that differentiates physical exercises and any sports, implying differences in the samples and the responses to stressors.

\subsection{Experimental Protocols}

A set of capped polyethylene test tubes were provided to involved subjects with an identification for the salivary collection. Salivary withdrawals for each involved subject were taken without any external stimulation directly and spontaneously deposited on the test tubes. The subjects were requested to refrain from eating and drinking for at least $2 \mathrm{~h}$ before sample collection [16]. Three withdrawals (in the morning, before lunchtime and in the evening) were collected in days without performances and in sedentary subjects. Four withdrawals were carried out the day of the trials/competitions (in the morning, closely before and after the performances and in the evening).

\subsection{Sample Storage}

Salivary samples were kept by volunteers in the home freezer and at $-20^{\circ} \mathrm{C}$ in laboratory, although salivary cortisol has a long-term stability. Samples were thawed and centrifuged at $4000 \mathrm{~g}$ for 15 minutes before being processed in the measurement apparatus testing cortisol concentration. Salivary mucus is fragmented and remains residual in the bottom of the tube after the centrifugation; its volume does not contribute to the evaluation of the concentration. In order to allow a proper comparison of the cortisol trends, all the figures adopt the same scales for the vertical axis.

\subsection{Sample Analyses}

The Abbott AxSYM Auto-analyzer apparatus was employed for the analyses of the whole set of samples. The Axym system performs an immunological analysis to measure automatically the cortisol concentration in presence of proper reagents. The apparatus can process up to 80 - 120 samples contemporarily by adopting the FPIA methodology, i.e. the fluorescence immunological dosage with polarized light, a method suitable to investigate low molecular weight samples as cortisol (molecular weight 362 Dalton). The experimental data provided by the above apparatus are analyzed and reported throughout the graphical software. It is a well-known result that different experimental techniques leads to different experimental values in particular in connection with scaling factors. These effects do not affect the evaluation of the concentration trends and of their slopes. Thus, it needs to be remarked that all the results reported in the present paper were obtained consistently with the same apparatus and the same procedures; experimental errors never exceed 5\% with respect to Abbott's reference samples. 
Further, too small salivary samples were excluded from the present analyses in order to preserve the same experimental error in all the reported measurements.

\section{Results}

\subsection{Cortisol Trend in Days without Winter Swimming Trials and Sports Competitions}

In Figure 1(a) the concentration of salivary cortisol measured in sedentary subjects is reported, selected to show the extreme upper and lower behaviors observed. Absolute values from $0.5(\mu \mathrm{g} / \mathrm{dl})$ in the morning down to 0.2 $(\mu \mathrm{g} / \mathrm{dl})$ in the evening are reported, in agreement with the expected circadian trend reported in the literature [17]. A mean slope of -0.06 c.u./h (defining $\mu \mathrm{g} / \mathrm{dl}$ as current concentration units) is obtained up to the evening starting from hours corresponding to the competitions or trials reported below.

In Figure 1(b) the comparison of cortisol trend in salivary samples from eleven winter swimmers collected in a day without any trials is reported. Absolute cortisol values are in the range $2.8-0.8(\mu \mathrm{g} / \mathrm{dl})$ in the morning and $2.7-0.3(\mu \mathrm{g} / \mathrm{dl})$ in the evening.

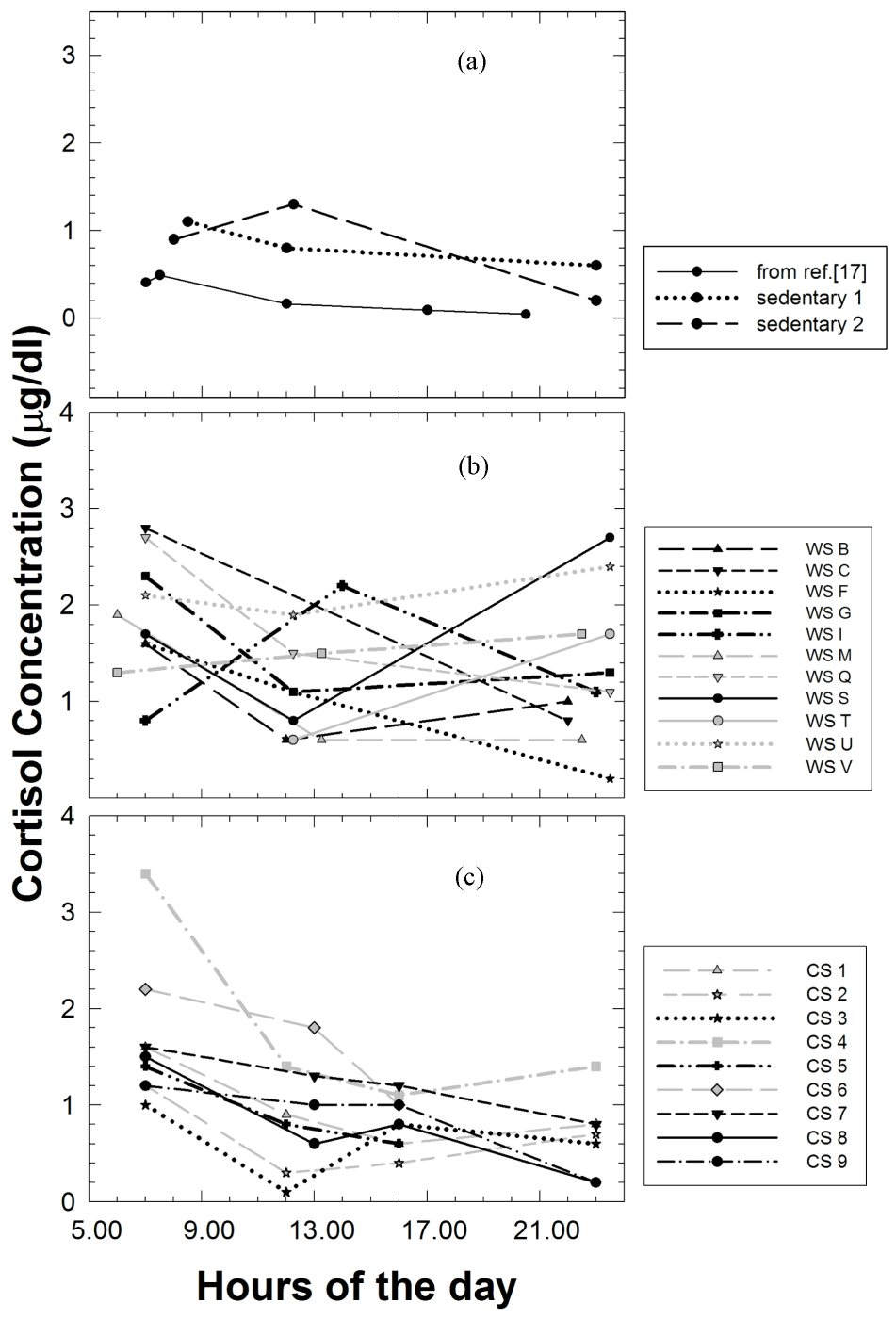

Figure 1. Comparisons of circadian cortisol behavior in salivary samples. (a) Results in sedentary volunteers (dot and broken lines) and from literature data (solid line) [17] (Salimetrics Web site); (b) In eleven winter swimmers (WS) collected in a day without any trials; (c) In nine canoe and canoe-polo sportsmen (CS) collected in a day without any competitions. 
In Figure 1(c) the comparison of cortisol trend in salivary samples from nine sportsmen collected in a day without any canoe and canoe-polo competitions is reported. Absolute cortisol values are in the range 3.5 - 1.0 $(\mu \mathrm{g} / \mathrm{dl})$ in the morning and $1.3-0.2(\mu \mathrm{g} / \mathrm{dl})$ in the evening.

Comparing the cortisol trends concerning results in a day without trials (Figure 1(b)) and competitions (Figure 1(c)) with the circadian rhythm reported in Figure 1(a), it can be observed that in a day without trials and competitions, the cortisol trends of the sportsmen resemble the normal circadian rhythm, whereas winter swimmers show a deviation from the normal pattern.

\subsection{Cortisol Trend in Days with Winter Swimming Trials and Sport Competitions}

In Figure 2(a) and Figure 2(b) the cortisol concentration in winter swimmers collected in the days with trials is reported. As can be seen, abrupt concentration changes are detected at the time of the trials, surprisingly both increasing than decreasing absolute concentration values are detected. Moreover, values of cortisol concentration remain fairly elevated in the evening. Cortisol absolute concentrations are shown to assume values in a range between $3.4-0.6(\mu \mathrm{g} / \mathrm{dl})$ (morning) and 2.6 - $0.4(\mu \mathrm{g} / \mathrm{dl})$ (evening) in the first trial and 2.9 - $0.7(\mu \mathrm{g} / \mathrm{dl})$ (morning) and $2.0-0.0(\mu \mathrm{g} / \mathrm{dl})$ (evening) in the second trial.

In Figure 3 the cortisol concentration in sportsmen competitions collected in a day with competition is reported. As can be seen, abrupt concentration changes are detected at the time of the competition, both increasing than decreasing absolute concentration values are detected similarly to the group of winters swimmers. Moreover, absolute values of cortisol concentration remain fairly elevated in the evening. The same results were obtained from a second day of competition (data not shown). Absolute cortisol values are shown to assume values in a range $3.3-0.4(\mu \mathrm{g} / \mathrm{dl})$ in the morning and $2.4-0.5(\mu \mathrm{g} / \mathrm{dl})$ in the evening.

\section{Discussions}

Other studies pinpointed salivary cortisol as the hormone index responsible for the activity of the neuroendo-

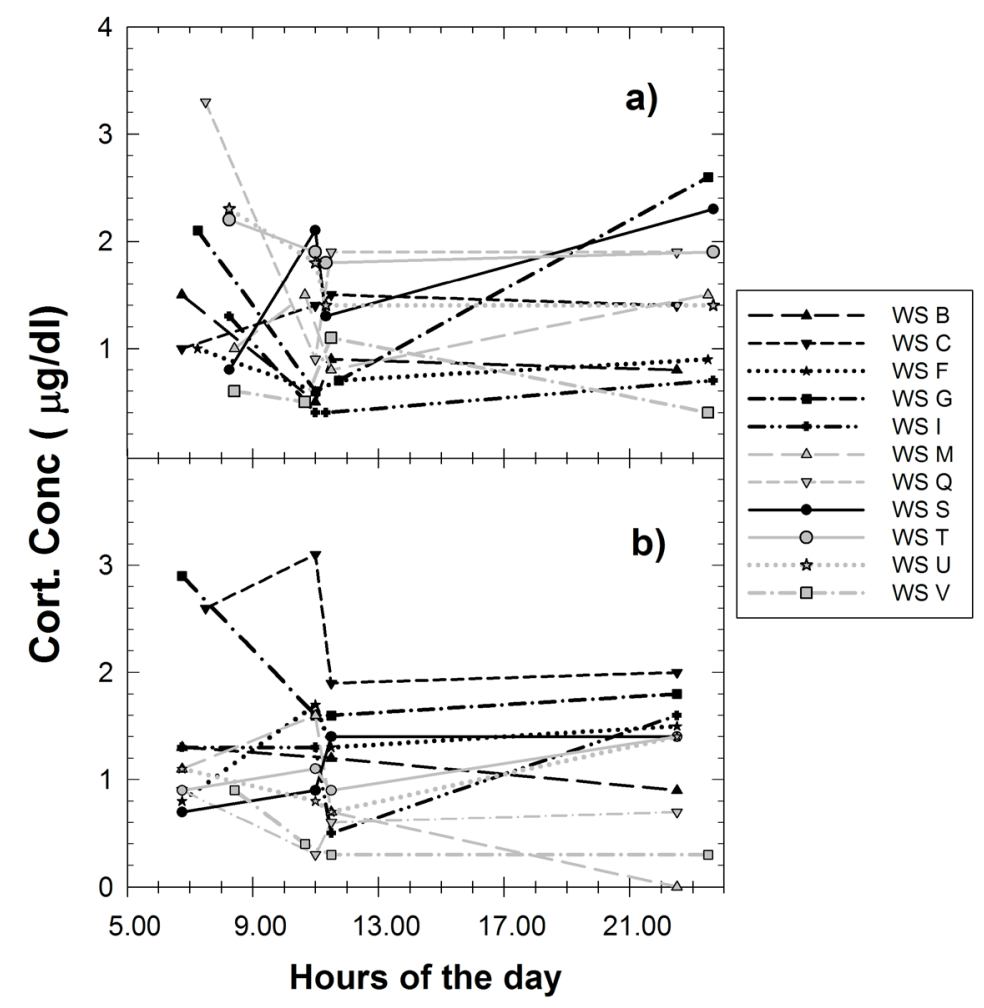

Figure 2. Comparisons of circadian cortisol behavior in salivary samples in the first (a) and second (b) day of trials in eleven (B - V) winter swimmers (WS). 


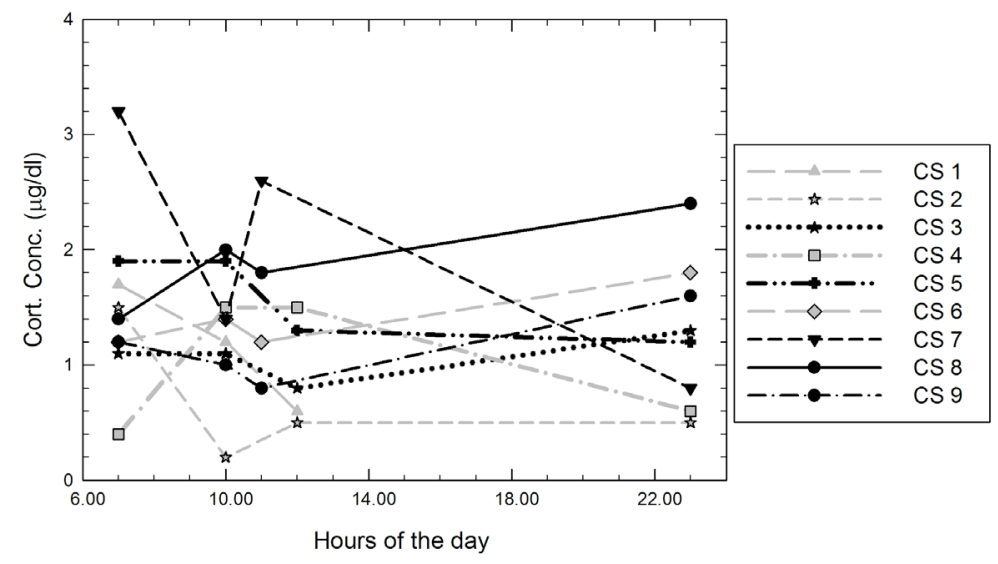

Figure 3. Comparisons of circadian cortisol behavior in salivary samples in nine (1 - 9) canoe and canoe-polo sportsmen (CS).

crine axis when it is sped up from several stressor performances or physical efforts [6] [7].

Salivary cortisol shows an irregular physiological trend along a day, but it follows a global tendency to decrease its concentration from the morning till the evening (the circadian rhythm) [18] [19] and to increase during the night to return to high morning values. In case the organism is sped up from unexpected performances, or from physical efforts, a noticeable adaptation [11] resulting in an abrupt variation of cortisol levels is determined with respect to the circadian rhythm [20].

Four literature observations ranging from the positive to the very negative can be envisaged in the medical literature and in the present work results concerning extreme exercises:

1) Positive stress (eustress) results from physiological adaptation of organisms to sport and also to extreme trials i.e. the reactivity of the organism is improved [21]-[23].

2) In winter swimmers, not physiological levels of cortisol in the evening are measured as shown in Figure 1(b) with respect to the normal circadian rhythm in Figure 1 (a) $(0.6-0.2 \mu \mathrm{g} / \mathrm{dl})$, both in days with $(2.6-0.0 \mu \mathrm{g} / \mathrm{dl})$ and without trials $(2.7-0.3 \mu \mathrm{g} / \mathrm{dl})$. Such concentrations must be regarded as subclinical, whereas larger evening concentrations are well known signals of Cushing, diabetes dismetabolic pathologies [20] and cardiovascular [23] risks.

3) Extreme stress in winter swimmers trials can lead in some cases to detrimental conditions [24] and to the death in accidental immersions [25] [26].

4) The amount of variations of cortisol concentration after sea-swimming in subjects used to the winter swimming practice decreases at repeating the number of trials. In other words, as observed by Leppaluoto $\mathrm{J}$ et al. and Hooper et al., subjects well trained to winter swimming, and thus to face extreme stressors, show a lower neuroendocrine adaptation reaction with respect to occasional practitioners [27] [28].

Results of the present study are expected to describe effectively the activity of the neuroendocrine axis during the sport exercise (like the winter swimming) as well as in other sport tests (canoes and canoe-pole) since the cortisol concentration can be readily evaluated through easy and repeated salivary withdrawals. Biochemical evidences might confirm or exclude any claimed benefits to health at the bases of the self-prescription practise adopted by winter swimmers to improve their feeling of well-being and enjoying advantages similar to positive stress, both in days with and without performances.

\subsection{Behavior in Days without Trials and Competitions}

Comparing the data obtained on the canoeists and on the canoe-pole sportsmen with those carried out on the winter swimmers, the trend of the cortisol in the days without performances appears to undergo the following variations:

1) a faster recovery of the circadian low level is achieved in the evening hours by sportsmen

2) the winter swimmers experience an increasing or a constant trend, deviating significantly from the circadian decrease in the evening

3) the data of winter swimmers are characterized by high cortisol absolute values, signing a difficulty to 
re-establish the physiological levels of cortisol.

These results turn out unexpected since the volunteers were selected on the basis of their well-being state checked by anamnestic-clinical procedure.

As shown by the histogram in Figure 4(a), the $55 \%$ of sportsmen experience a cortisol decrease towards the

evening; in the $45 \%$ sportsmen cortisol increases in the

evening, although with a small mean slope of +0.03 c.u./h with respect to the corresponding circadian slope of -0.06 c.u./h defined above. Absolute values of cortisol remain below $1.5 \mu \mathrm{g} / \mathrm{dl}$ in sportsmen as shown in Figure 1 (c).

The difficulty of winter swimmers to restore the circadian rhythm is made clear by the low percentage of $20 \%$ of winter swimmers showing a circadian trend restoration as shown in Figure 4(a); by the large absolute values in the range $2.5-1.5 \mu \mathrm{g} / \mathrm{dl}$ and by the large positive slopes up to +0.20 c.u./h as shown in Figure 1(b).

As discussed above, these days without trials occur in the middle of a week between two swimming trials in the winter season, and also in the middle of the many trials experienced in the whole season. Thus, this rather surprising result might be explained by the persisting influence of the extreme stressor, larger than in the competitive canoe sports. A better understanding would be provided by comparisons with similar observations in other groups of subjects experiencing extreme thermal stresses.
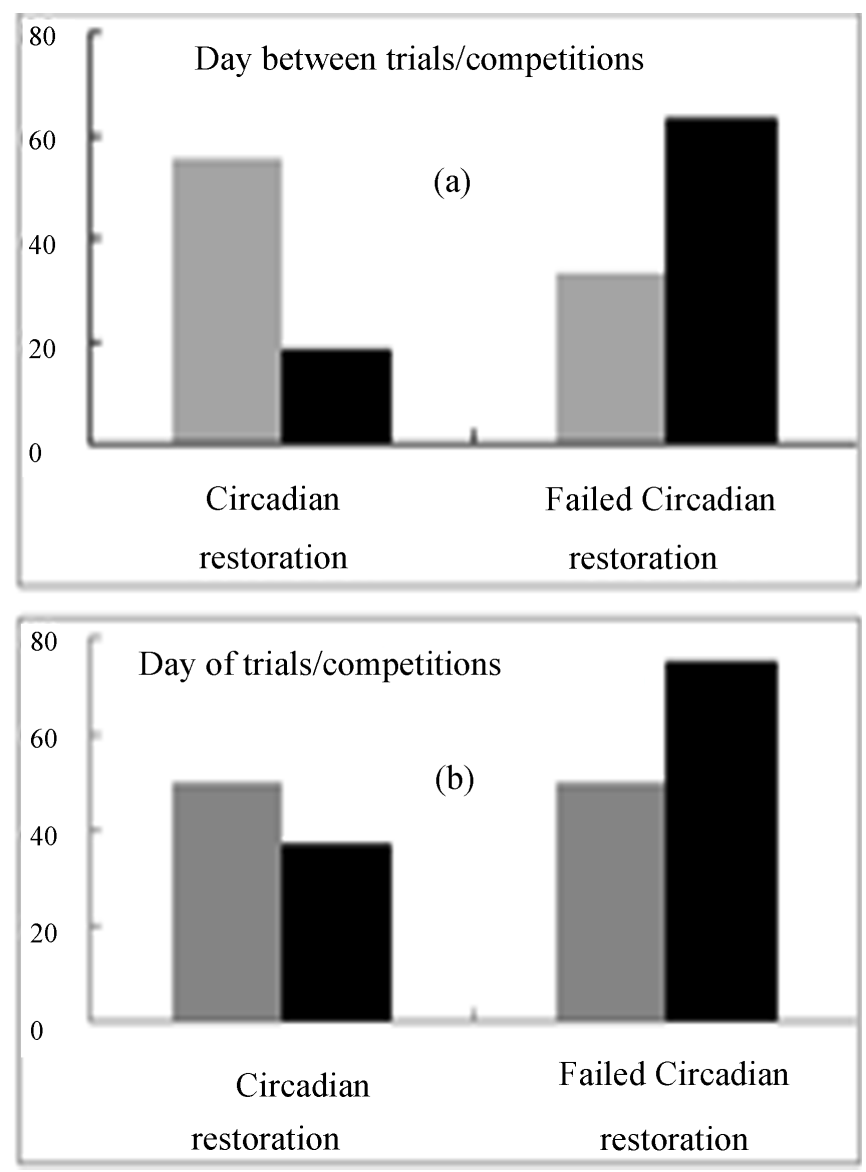

Figure 4. (a) Percentage of sportsmen (grey bars) and winter swimmers (black bars) that recover/fail to recover the circadian cortisol concentration in days without but between two performances. The majority of sportsman recover the circadian cortisol level in the evening whereas winter swimmers are shown to follow the opposite trend; (b) Percentage of sportsmen (grey bars) and winter swimmers (black bars) that recover/fail to recover the circadian cortisol concentration in days of performances from the moment of trials/competitions up to the evening. 


\subsection{Behavior in Days with Trials or Competitions}

The cortisol behavior in sport competition or trials days shows basically similar features in the two categories investigated as shown in Figure 2(a) and Figure 2(b) and Figure 3. The 50\% of sportsmen and $40 \%$ of winter swimmers is shown to restore the circadian cortisol concentration or to remain almost constant (i.e. slopes above +0.01 c.u./h) after the trials/competitions up to the evening of the same day of the performances. Percentages reported in Figure 4(b) are obtained including the results of two events. In winter swimmers, cortisol concentration increases occur systematically with higher probability than decreasing cases. In sportsmen, there are no evidences of a systematic trend.

\subsection{Behavior in Days with Trials or Competitions at the Time of the Performances}

At the time of the competitions, results of sportsmen show variations of the cortisol levels as shown by the percentage reported in Figure 5. In the first competition, six out of nine sportsmen experience an abrupt variation of cortisol ranging from -1.2 c.u./h up to +1.7 c.u./h. Two sportsmen show a fast increase and no significant variation is detected in one sportsman. In the second competition, six sportsmen out of nine show a fast increase of cortisol, three a fast decrease. Four sportsmen examined behave in the same way in the two competitions.

At the time of the trials, winter swimmers also show variations of the cortisol levels. As reported in Figure 5, an abrupt increase of cortisol is observed in six and a similar fast decrease in four subjects; in one subject no appreciable cortisol variation is observed. In the second winter swimming trial an abrupt increase of cortisol is observed in two subjects, a decrease in seven and no variation in only one subject is observed. Four winter swimmers out of eleven among the examined subjects behave with the same trends in the two trials. The slopes at the time of the trials range from -2.7 up to +2.0 c.u./h.

Substantially, sportsmen and winter swimmers in the moment of the performances behave with similar abrupt variations of cortisol concentration, although differently in the first with respect to the second performance. The abrupt variations at the time of the events in the two examined categories strengthen the explanation in terms of an adaptation syndrome.

However it is worth to emphasize that the age of the winter swimmers has a larger variability (from 19 to the 86 years) with respect to the sportsmen, and also a higher mean age.

A stressor coefficient can be assumed as the geometrical average of the maximum slopes (absolute values): winter swimmers and sportsmen competitions give 2.4 and -1.4 c.u./h, respectively.

It turns out that these stressor coefficients can represent quantitatively the neuroendocrine adaptation response of the organism to external performances. On the other hand, it turns out unpractical as far as the sign of the slope is concerned, to adopt cortisol detection to preview the sign of the variation. The much larger stressor coefficient in winter swimming trials and also possibly the wide range of age can explain the long term persistence of high cortisol in days without trials discussed above.

Being minimal the percentage of subjects showing no significant variations of cortisol in the moment of the performance (as shown in Figure 5), constant the tendency to reverse the behavior and also constant the number of individuals that behave in the same way within the two groups (lower than $50 \%$ of the respective populations), it can be concluded from these results that cortisol cannot be regarded as the preeminent parameter to assess in

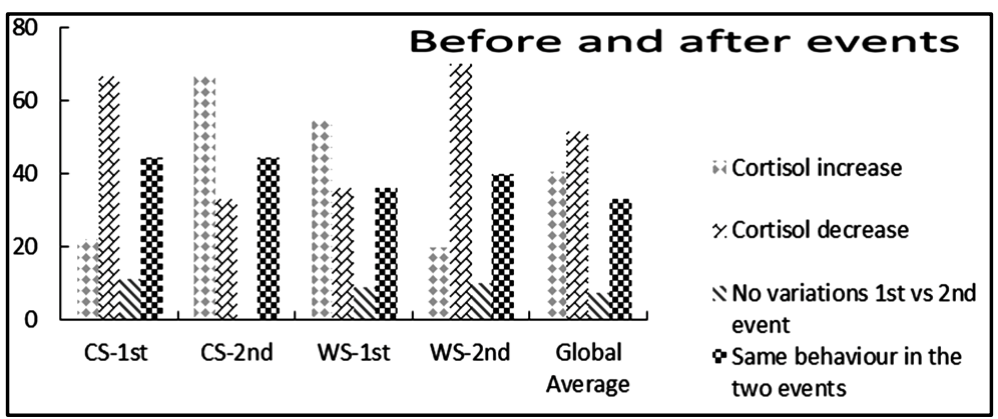

Figure 5. Percentage of sportsmen and winter swimmers that behave accordingly to the four indicated conditions relatively to the cortisol concentration variations before and after the performances. 
every aspects the activity of the neuroendocrine axis in proximity of the sport contest or of the physical exercise. Thus, other endocrine mechanisms are involved [29] and further investigations are deserved.

\subsection{Search of Correlations}

In this section, the correlation of cortisol as a neuroendocrine response to a positive stress at the moment of the trials and/or competitions is investigated with respect to cortisol evening levels both in days with and without winter swimming trials or canoe competitions. Correlations with cortisol levels in the morning were also investigated, showing no significant evidences in both sportsmen and winter swimmers. The correlations reported in Figure 6(a) are related to sportsmen (grey bars) and winter swimmers (black bars) that decrease (down) cortisol after the competitions/trials and that maintain high/low cortisol levels in the evening of both days with (P) and between performances (BP).

In Figure 6(b) the correlations are reported with respect to the complementary behavior after the competitions/trials, i.e. the cortisol increase (up). Main results arise from the more significant differences of correlations:

1) Sportsmen correlate with high probability the decrease (down) and with low probability the increase (up) with respect to low levels in the evening of days between performances and of the performances, irrespectively of the up-down conditions after the competitions;

2) Winter swimmers correlate with high probability up and down vs. high levels both in the days with and between trials;

3) Winter swimmers show a tendency to decrease cortisol at the time of the trial in particular in those who maintain a high level in the evening on the day of the trials, as reported in Figure 6(a);

4) In the day between performances, sportsmen show low levels of cortisol also in the evening, as the sedentary subjects (see Figure 1(a)) but differently from the winter swimmers. Low evening cortisol levels correspond to the physiological behavior of the circadian cortisol.

The Fisher's exact test applied to the contingency table corresponding to the data in the histogram in Figure 6(a) of the down and high, down and low observations gives a significance level of $88 \%$ with respect to the more extreme tables.

\section{Concluding Remarks and Forthcoming Plannings}

In the present study, the behavior of cortisol was examined in view of its relationships with the activity of the neuroendocrine axis, in the sportsmen and in winter swimmers as representative of persons taking part to "sport-like exercise”. Moreover, as discussed above, winter swimmers claim that they self-prescribe such exercise to enjoy similar benefits with respect to positive stress both in days with and without performances. Present work results point out the different behavior in the two groups regarding the physiological re-establishment of the values of cortisol in the evening of days without performances. Namely:

1) The cortisol behavior in the winter swimmers exhibits an analogous syndrome of adaptation at the time of the trial event and, inter alia, without the psychical pressure of the competition although characterized by extreme thermal stressor.

2) It appears clearly that winter swimmers experience a difficult physiological re-establishment of cortisol circadian levels in the evening, in particular in day without trials.

3) Almost regularly, elevated absolute values of cortisol are detected.

It must be taken into account that, in the moment of the winter swimmers trials, the cold temperatures of sea water $\left(8^{\circ} \mathrm{C}-14^{\circ} \mathrm{C}\right)$ require the organism to undergo a thermo genic recovery [8] that might affect the cortisol behavior and the neuroendocrine activity over an extended period with respect to the sport competitions examined. This hypothesis is corroborated by the stressor coefficient values reported above.

Therefore, further studies are planned to confirm the above results, mainly in view of the growing relevance and the widespread diffusion of uncompetitive sport-like exercises in the population, even marked by extreme stressors. Further, winter swimmers claim to choose such exercise to exploit the claimed benefits from the specific adaptation to an extreme stressor such as cold water in wintertime. The clinical interest of the present study in fact can be put in relation with the growing inclination to the self-prescription by the person who trusts in a "healthy life style" without to fulfill adequate clinical/ medical checks. Differently, sportsmen can generally trust 

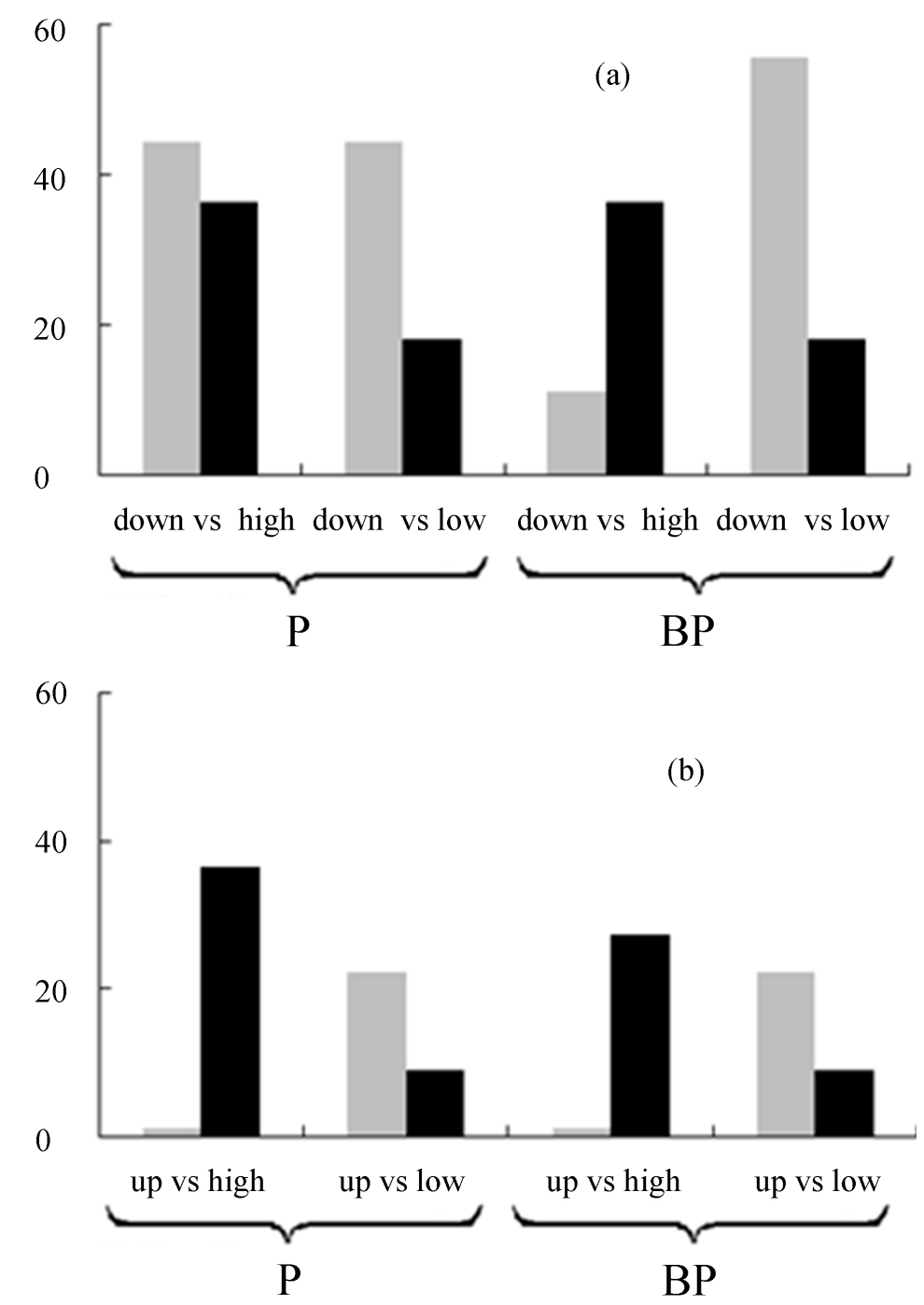

\begin{abstract}
Figure 6. Correlation of cortisol variations after the winter swimmers trials (black) and sportsmen competitions (grey) with respect to the cortisol levels observed in the evenings. First two couples of columns regard the correlation with evenings of the same trials/competitions days (grouped under the symbol P), the last two couples of columns are related to evenings in days without but between trials or competitions (symbol BP). (a) Correlations of decrease trend (down) at the time of the events with respect to evening values; (b) Correlations of increase trend (up) at the time of the events with respect to evening values.
\end{abstract}

on the attendance of sport doctors teams prepared to supply accurate controls as regards their physical conditions in days of competitions and in others, besides to assess periodically their fitness to practice sports.

Thus, in agreement with the precautions suggested by Hipp et al. [29] and by Bleakley et al. [30], the results strengthen the view that sport-like exercise as the winter swimming, particularly when carried out in extreme conditions, may well pose some treats ranging from subclinical aspects up to dismetabolic pathologies and even cardiovascular risks, although excluded in the volunteers selected in the present investigation. The need of further clinical studies correlated with biochemical data is pointed out to ascertain about the adaptation syndrome evidenced by present work results. Thus, in agreement with literature findings, adrenal response to neuroendocrine activity suggests that a doctor might verify the physiological and pathological conditions of the patient willing to face for the first time a sport exercise with extreme stressors. 


\section{Acknowledgements}

The authors would like to thank Dr. A. Cioè, Dr. D. Chiapperini, and Dr. S. Bertamini of Dipartimento di Scienze della Salute University of Genoa and to Associazione Nuotatori del Tempo Avverso and the collaboration of Abbott s.r.l.

\section{References}

[1] Schulz, P., Kirschbaum, C., Prussner, J. and Hellhammer, D. (1998) Increased Free Cortisol Secretion after Awakening in Chronically Stressed Individuals Due to Work Overload. Stress Medicine, 14, 91-97. http://dx.doi.org/10.1002/(SICI)1099-1700(199804)14:2<91::AID-SMI765>3.0.CO;2-S

[2] Izawa, S., Shirotsuki, K., Sugaya, N., Ogawa, N., Suzuki, K. and Nomura, S. (2007) The Application of Saliva to an Assessment of Stress: Procedures for Collecting and Analyzing Saliva and Characteristics of Salivary Substances. Japanese Journal of Complementary and Alternative Medicine, 4, 91-101. http://dx.doi.org/10.1625/jcam.4.91

[3] Hellhammer, D.H., Wüst, S. and Kudielka, B.M. (2009) Salivary Cortisol as a Biomarker in Stress Research. Psychoneuroendocrinology, 34, 163-171. http://dx.doi.org/10.1016/j.psyneuen.2008.10.026

[4] O’Connor, J.P. and Corrigan, D.L. (1987) Influence of Short-Term Cycling on Salivary Cortisol Levels. Medicine \& Science in Sports \& Exercise, 19, 224-228.

[5] Lombardi, G., Ricci, C. and Banfi, G. (2011) Effect of Winter Swimming on Haematological Parameters. Biochemia Medica, 2, 71-78. http://dx.doi.org/10.11613/BM.2011.014

[6] Fukuda, S. and Morimoto, K. (2011) Lifestyle, Stress and Cortisol Response: Review I. Environmental Health and Preventive Medicine, 6, 9-14. http://dx.doi.org/10.1007/BF02897303

[7] Fukuda, S. and Morimoto, K. (2011) Lifestyle, Stress and Cortisol Response: Review II. Environmental Health and Preventive Medicine, 6, 15-21. http://dx.doi.org/10.1007/BF02897304

[8] De Weerth, C., Jansen, J., Vos, M.H., Maitimu, I. and Lentjes, E.G.W.M. (2007) A New Device for Collecting Saliva for Cortisol Determination. Psychoneuroendocrinology, 32, 1144-1148. http://dx.doi.org/10.1016/j.psyneuen.2007.07.005

[9] Aardal, E. and Holm, A.C. (1995) Cortisol in Saliva-Reference Ranges and Relation to Cortisol in Serum. European Journal of Clinical Chemistry and Clinical Biochemistry, 33, 927-932.

[10] Young, E.A. and Nolen-Hoeksema, S. (2001) Effect of Ruminations on the Saliva Cortisol Response to a Social Stressor. Psychoneuroendocrinology, 26, 319-329. http://dx.doi.org/10.1016/S0306-4530(00)00059-7

[11] Del-Corral, P., Mahon, A.D., Duncan, G.E., Howe, G.E. and Craig, B.W. (1994) The Effect of Exercise on Serum and Salivary Cortisol in Male Children. Medicine \& Science in Sports \& Exercise, 26, 1297-1301.

[12] Dahlgren, A., Kecklund, G., Theorell, T. and Akerstedt, T. (2009) Day-to-Day Variation in Saliva Cortisol—Relation with Sleep, Stress and Self-Rated Health. Biological Psychology, 82, 149-155. http://dx.doi.org/10.1016/j.biopsycho.2009.07.001

[13] Watson, A.W.S. (1984) The Physique of Sportsmen: A Study Using Factor Analysis. Medicine \& Science in Sports \& Exercise, 16, 287-293. http://dx.doi.org/10.1249/00005768-198406000-00016

[14] Smilios, I., Pilianidis, T., Karamouzis, M. and Tokmakidis, S.P. (2003) Hormonal Responses after Various Resistance Exercise Protocols. Medicine \& Science in Sports \& Exercise, 35, 644-654. http://dx.doi.org/10.1249/01.MSS.0000058366.04460.5F

[15] Harriss, D.J. and Atkinson, G. (2011) Update-Ethical Standards in Sport and Exercise Science Research. International Journal of Sports Medicine, 32, 819-821. http://dx.doi.org/10.1055/s-0031-1287829

[16] Toda, M., Morimoto, K., Nagasawa, S. and Kitamura, K. (2004) Effect of Snack Eating on Sensitive Salivary Stress Markers Cortisol and Chromogranin A. Environmental Health and Preventive Medicine, 9, 27-29. http://dx.doi.org/10.1265/ehpm.9.27

[17] Salimetrics Web Site (2013) State College, PA 16803 USA. http://www.salimetrics.com/documents/1-3002.pdf

[18] Matsuda, S., Yamaguchi, T., Okada, K., Gotouda, A. and Mikami, S. (2012) Day-to-Day Variations in Salivary Cortisol Measurements. Journal of Prosthodontic Research, 56, 37-41. http://dx.doi.org/10.1016/j.jpor.2011.04.004

[19] Hellhammer, J., Fries, E., Schweisthal, O.W., Schlotz, W., Stone, A.A. and Hagemann, D. (2007) Several Daily Measurements Are Necessary to Reliably Assess the Cortisol Rise after Awakening: State- and Trait Components. Psychoneuroendocrinology, 32, 80-86. http://dx.doi.org/10.1016/j.psyneuen.2006.10.005

[20] O’Donnell, K., Badrick, E., Kumari, M. and Steptoe, A. (2008) Psychological Coping Styles and Cortisol over the Day in Healthy Older Adults. Psychoneuroendocrinology, 33, 601-611. http://dx.doi.org/10.1016/j.psyneuen.2008.01.015 
[21] Simenckova, M., Jansky, L., Lesna, I., Vybiral, S. and Sramek, P. (2000) Role of Beta Adrenoceptors in Metabolic and Cardiovascular Responses of Cold Exposed Humans. Journal of Thermal Biology, 25, 437-442. http://dx.doi.org/10.1016/S0306-4565(00)00007-3

[22] Bittel, J. (1992) The Different Types of General Cold Adaptation in Man. International Journal of Sports Medicine, 13, S172-S176. http://dx.doi.org/10.1055/s-2007-1024630

[23] Wright, C.E., O’Donnell, K., Brydon, L., Wardle, J. and Steptoe, A. (2007) Family History of Cardiovascular Disease Is Associated with Cardiovascular Responses to Stress in Healthy Young Men and Women. International Journal of Psychophysiology, 63, 275-282. http://dx.doi.org/10.1016/j.ijpsycho.2006.11.005

[24] Kolettis, T.M. and Kolettis, M.T. (2003) Winter Swimming: Healthy or Hazardous? Evidence and Hypotheses. Medical Hypotheses, 61, 654-656. http://dx.doi.org/10.1016/S0306-9877(03)00270-6

[25] The Eurowinter Group (1997) Cold Exposure and Winter Mortality from Ischaemic Heart Disease, Cerebrovascular Disease, Respiratory Disease, and All Causes in Warm and Cold Regions of Europe. Lancet, 349, 1341-1346. http://dx.doi.org/10.1016/S0140-6736(96)12338-2

[26] Ducharme, M.B. and Lounsbury, D.S. (2007) Self-Rescue Swimming in Cold Water: The Latest Advice. Applied Physiology, Nutrition, and Metabolism, 32, 799-807. http://dx.doi.org/10.1139/H07-042

[27] Leppaluoto, J., Westerlund, T., Huttunen, P., Oksa, J., Smolander, J., Dugué, B. and Mikkelsson, M. (2008) Effects of LongTerm Whole-Body Cold Exposures on Plasma Concentration of ACTH, Beta-Endorphin, Cortisol, Catecholamines and Cytokines in Healthy Females. Scandinavian Journal of Clinical \& Laboratory Investigation, 68, 145-153. http://dx.doi.org/10.1080/00365510701516350

[28] Hooper, S.L., Mackinnon, L.T., Gordon, R.D. and Bachmann, A.W. (1993) Hormonal Responses of Elite Swimmers to Overtraining. Medicine and Science in Sports and Exercise, 25, 741-747.

[29] Hipp, A.A., Heitkamp, H.C., Röcker, K. and Dickhuth, H.H. (2004) Hypertrophic Cardiomyopathy-Sports-Related Aspects of Diagnosis, Therapy, and Sports Eligibility. International Journal of Sports Medicine, 25, 20-26. http://dx.doi.org/10.1055/s-2003-45227

[30] Bleakley, C.M. and Davison, G.W. (2010) What Is the Biochemical and Physiological Rationale for Using Cold-Water Immersion in Sports Recovery? A Systematic Review. British Journal of Sports Medicine, 44, 179-187. http://dx.doi.org/10.1136/bjsm.2009.065565 